\title{
Gestión de la Madurez Tecnológica Nivel 1 (TRL 1); Estrategia de Gestión de la Innovación de Producto en Procesos de Diseño Mecánico
}

\author{
Tlapanco Rios, Ernesto Isaac ${ }^{1, *}$; ; Castaño Urrego, Carlos Andrés 2 (iD) \\ ${ }^{1}$ Departamento de Estudios Multidisciplinarios Sede Yuriria, División de Ingenierías, Campus Irapuato-Salamanca, \\ Universidad de Guanajuato, México \\ ${ }^{2}$ Universidad SantoTomás, Facultad de Ingeniería Mecánica, Bogotá, Colombia
}

\begin{abstract}
Resumen: Desde el punto de vista de la gestión tecnológica, la formulación de estrategias es la actividad clave para un líder de proyecto además de proponer estrategias evaluables, validadas y repetibles. El líder del proyecto además, debe justificar sus estrategias con respecto a los sistemas de innovación como puede ser el uso de los niveles de maduración de la tecnología (TRL) para que los departamentos de ingeniería, financiero y mercadotecnia gestionen los recursos para cumplir con las actividades solicitadas. Esta investigación muestra evidencia del tipo de actividades estratégicas que genera un departamento de ingeniería para la innovación de producto, específicamente en el diseño y estándares de madurez tecnológica. Parte de visualizar el cumplimiento de una especificación TRL 1, seguido de demostrar por medios de análisis de ingeniería mecánica el cómo se puede estandarizar eficientemente un proceso analítico de corte de material con métodos de elemento finito y la ayuda del CAE para convertirlo en un proceso que permita certidumbre en procesos no comunes ni considerados en el diseño mecánico, el resultado puede resultar innovador para la enseñanza de la ingeniería mecánica e incluso para la manufactura del troquelado si se cuentan con las herramientas de cómputo o alianzas con centros de investigación y desarrollo.
\end{abstract}

Palabras clave: Gestión, Innovación, Ingeniería, Tecnología, CAE.

\section{Management of Technology Readiness Level 1 (TRL 1); Product Innovation Management Strategy in Mechanical Design Processes}

\begin{abstract}
From the point of view of technological management, the strategy is the key for a project leader in addition to evaluable validated and repeatable strategies. The project leader must also justify its strategies regarding innovation systems, such as the use of Technology Readiness Level (TRL) for the engineering, financial and marketing departments. This research gives evidence of the strategic activities generated by the engineering department focused on product innovation, specifically in the design process and technological maturity. This research shows evidence of the type of strategic activities by an engineering department for product innovation, specifically in the design and tecnological standards. It begins focusing on compliance TRL 1 specification, proving by mechanical engineering analysis how to efficiently standardize a die cutting with finite element methods (CAE) developing a process that allows certainty in die cutting that is not common or considered in mechanical design, the result can be innovative for the teaching of mechanical engineering and even for the manufacture of die cutting if there are computer tools or alliances with research and development centers.
\end{abstract}

Keywords: Management, Innovation, Engineering, Technology, CAE.

\section{INTRODUCCIÓN}

La gestión de la tecnología en la sociedad es el resultado de la articulación de los agentes empresariales vinculados con agentes académicos y gubernamentales, es decir, es un mecanismo que articula a los sistemas de innovación (Hidalgo, 2006).
El agente empresarial que lidera la gestión tecnológica, lo hace a través de modelos de negocio tecnológico y estrategias comparativas, para desarrollar nuevos productos, es decir, los modelos de negocio tecnológico aplican una estrategia de mejora tecnológica para el lanzamiento de nuevos productos, creando mercado o incrementando su participación dentro del mercado en que se encuentran. 
Dentro de la estrategia tecnológica, una de los enfoques más importantes del valor de las invenciones de un modelo de negocio tecnológico, es el registro de la propiedad industrial, específicamente patentes y modelos de utilidad. Que permite mantener la participación en el mercado mientras la invención sea comercializada (Garcia y Münch, 1992).

Otro agente de la transferencia tecnológica es la academia, la cual procura avalar una innovación por medio del desarrollo metódico del producto, para lo cual normaliza los niveles de madurez tecnológica (Technology Readiness Level -TRL-) concepto que nace en la National Aeronautics and Space Administration (NASA) y que México ha adoptado a través del Consejo Nacional de Ciencia y Tecnología (CONACyT), el instituto utiliza el sistema de TRL para ubicar los proyectos que recibe en las convocatorias de financiamiento (CONACyT, 2019), es importante destacar que el CONACyT ha tomado como referencia la interpretación del TRL del Ministerio de Industria, Comercio y Turismo (Aplicaciones de la Teoría de Juegos a la Economía Industrial, 2014).

Para fomentar el desarrollo tecnológico, el CONACyT agrupa los niveles del TRL en seis categorías, los TRL 1 y 2 forman la categoría de desarrollo de la invención, los TRL 3 y 4 forman la categoría de validación de concepto, el TRL 5 forma parte de la categoría de desarrollo de prototipo, los TRL 6 y 7 forman la categoría de producción piloto y demostración, el TRL 8 forma la categoría de introducción al mercado y el TRL 9 forma la categoría de expansión de mercado. Las seis categorías se muestran en la Tabla 2.

El modelo del CONACyT ha derivado la Norma Oficial Mexicana NMX-GT-004-IMNC-2012, Tabla 2, identifica cada nivel de TRL por medio de una lista de verificación con preguntas dicotómicas con respuestas de "si" o "no", lo que permite que la herramienta sea eficiente.

La categoría de desarrollo de la invención que incluye los niveles TRL 1 y 2, Figura 1, permite hacer una planeación estratégica en tres actividades principales:

- Innovación organizacional; induce a que el líder del proyecto organice un equipo de trabajo para el logro de los objetivos.

- Innovación de producto y transferencia de tecnología; define actividades claras como son;

a) investigación en publicaciones científicas para el desarrollo de ingeniería del producto o desarrollo de investigación básica,

b) búsqueda de patentes similares, lo que permite evaluar si es rentable la compra o renta de una patente o desarrollar la invención con fines de proteger la actividad industrial,

c) investigación del proceso de manufactura de la posible invención o en su caso la adaptación de tecnología existente.
- Innovación de mercadotecnia, define actividades para detectar si se adquiere tecnología existente y detectar posibles compradores de la invención tecnológica.

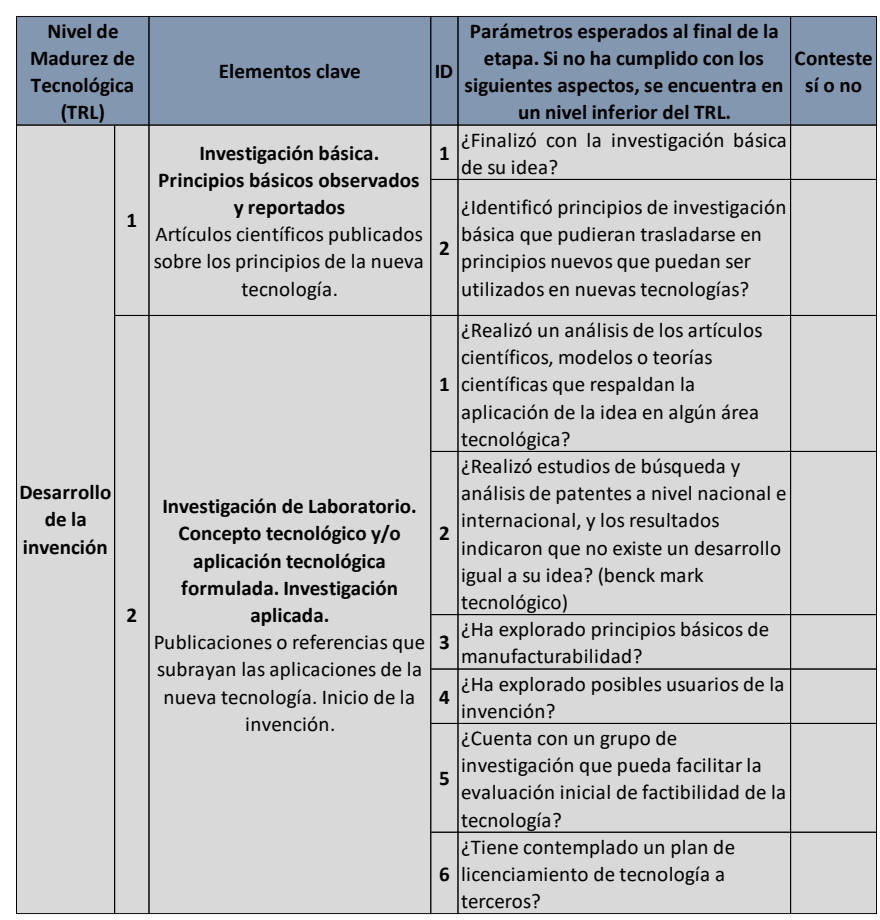

Figura 1. Categoría de desarrollo de la invención (TRL 1 y 2) de la Norma Oficial Mexicana NMX-GT-004-IMNC-2012 Fuente: CONACyT (2019)

Esta investigación se ha preparado con el fin de mostrar una memoria técnica útil para la estrategia de innovación de producto y transferencia de tecnología, es un desarrollo de ingeniería del producto, aplicado en procesos de diseño mecánico, del cuál se obtuvo una metodología para el diseño de troqueles de corte y que redujo costos en un $60 \%$, eliminando fases de pruebas para geometrías complejas de corte por medio de la optimización del diseño de punzones y matrices.

La metodología de diseño utilizada en esta estrategia de innovación de producto es el análisis de ingeniería por computadora (del inglés Computer Aided Engineering, CAE, en español la traducción es Ingeniería Asistida por Computadora).

\section{ESTRATEGIA DE INNOVACIÓN DE PRODUCTO EN PROCESOS DE DISEÑO MECÁNICO}

En la manufactura de procesos metalmecánicos de corte es común que una de las principales actividades sea el cambio de punzones o matrices, en ocasiones se le llama mantenimiento preventivo, sin embargo, no se permite trabajar a los herramentales hasta su máximo rendimiento debido a que se sobreexplota el mantenimiento preventivo y se hacen cambios antes de que sucedan las fallas dejando de explotar la vida útil de un herramental.

Por otra parte, en el caso de punzones y matrices se realiza un cambio preventivo para evitar la falla, descuidando que, una de las razones más importantes que previene la falla es el que un punzón y una matriz, se instalan sin comprobar su eficiencia 
contra el material que se troquela, es decir se toman decisiones sugeridas por los proveedores de herramentales a través de catálogos de productos sin comprobar la eficiencia de los productos por algún método experimental o de simulación o sin un análisis de falla.

El objetivo del caso que se presenta fue desarrollar un método de análisis CAE que complemente los análisis tradicionales de diseño e ingeniería del proceso de corte de una lámina de metal por medio de un punzón y una matriz, con el fin de comprobar que se puede utilizar confiablemente para la toma de decisiones de mantenimiento e incluso como propuesta de análisis CAE para diseño de punzones y matrices no convencionales.

La metodología es ajustar un método CAE por medio de otro método de ingeniería como es el análisis y diseño tradicionales, así se asegura que el método CAE sea confiable.

La geometría que se analiza en este estudio es convencional para los procesos de diseño en ingeniería, ya que geométricas complicadas requieren de una investigación que encuentre el modelo matemático idóneo para cada geometría.

La ventaja de ajustar un método CAE es que puede sustituir en ocasiones a la investigación de geometrías complicadas.

Nota: En caso de que el lector esté interesado en seguir la metodología propuesta, debe considerar que la metodología de este artículo solo es una sugerencia con un grado de confiablidad que permite la toma de decisiones en el diseño.

En esta investigación, se considera el estado lineal elástico de los materiales y no se consideran cambios de dureza a lo largo del material o imperfecciones, tampoco cambios de composición química que puede afectar abrasivamente al punzón o a la matriz, tampoco considera el uso de una prensa con pocas variaciones de carga y linealidad entre otros eventos que suceden en las áreas de manufactura.

Las propiedades de los materiales y las condiciones de frontera que se utilizan en este estudio son un material para laminar un acero AISI 1010, de naturaleza dúctil, el material de punzón y la matriz es un acero D2, templado con dureza de $58 \mathrm{HRC}$, de naturaleza frágil. Los valores de resistencia se encuentran declarados en Teicholz (1985, pág. 859) y Uddeholm (2003, pág. 3). Propiedades del material AISI 1010; módulo de elasticidad de 200,000 (MPa), módulo de Poisson 0.3, densidad $7.85 \times 10^{-6}\left(\mathrm{~kg} / \mathrm{mm}^{3}\right)$, coeficiente de expansión térmica $1.2 \times 10-5\left(1 /{ }^{\circ} \mathrm{C}\right)$, esfuerzo de fluencia a la tensión 300 $(\mathrm{MPa})$, esfuerzo último a la tensión $370(\mathrm{MPa})$, esfuerzo de fluencia a la compresión $300(\mathrm{MPa})$, esfuerzo último a la compresión $370(\mathrm{MPa})$. Propiedades del material D2; módulo de elasticidad 200,000 (MPa), módulo de Poisson 0.3, densidad $7.85 \times 10^{-6}\left(\mathrm{~kg} / \mathrm{mm}^{3}\right)$, coeficiente de expansión térmica $1.2 \times 10-51 /{ }^{\circ} \mathrm{C}$, esfuerzo de fluencia a la tensión 2,200 (MPa), esfuerzo último a la tensión 2,300 (MPa), esfuerzo de fluencia a la compresión 2,200 (MPa), esfuerzo último a la compresión 2,300 (MPa).
El por qué utilizar un modelado CAD-CAE lo explica de una forma razonable, Eric Teicholz en su libro CAD CAM Handbook (1985). 'La simulación. Muchos de los programas actuales tienen la ventaja de crear prototipos virtuales evitando así la fabricación de prototipos físicos que sirven para presentación del producto y para ciertos análisis estructurales, la mayoría de las veces cuando se presenta un prototipo físico, este ya es obsoleto y en lugar de convertirse en una herramienta confiable de desarrollo, es una pérdida de tiempo y dinero que muchas veces no justifica los resultados encontrados y por lo tanto no es un factor de mejora, en estos casos, un modelo virtual cumple con el $90 \%$ de las ventajas que un prototipo físico proporciona, además de incluir la ventaja de que puede desprender la misma información que un prototipo sin la inversión económica y tiempo ni hacerse obsoleto porque se puede actualizar en minutos".

\section{EVALUACIÓN DE ESFUERZOS DE UN PUNZÓN DE CORTE DE METAL EN PROCESOS METALMECÁNICOS DE TROQUELADO}

El problema analítico puede resolverse en cuatro pasos, el primer paso es formular el problema de corte de material para obtener el esfuerzo en que se vence el material a cortar, el segundo es formular el problema de compresión que se somete un punzón para conocer el esfuerzo máximo que puede soportar, el tercer paso es formular el problema de fatiga que se encuentra sometido el punzón para conocer el esfuerzo modificado por las cargas repetidas a las que se somete, y cuarto formular el problema de esfuerzos de contacto entre el punzón y el material a cortar.

El objetivo de formular los casos de esfuerzo es para conocer el valor mínimo que el punzón soporta ante el trabajo que realiza al cortar un material. Con el valor obtenido del menor esfuerzo que soporta el punzón, se puede contrastar contra el valor que se obtiene de un cálculo de CAE.

\subsection{Primer paso; formular el problema de corte de material para obtener el esfuerzo en que vence el material a cortar}

El modelo de corte que se calcula es para un material que es sometido a corte, con un punzón y matriz que entre ambos se coloca el material a cortar, véase Figura 2.

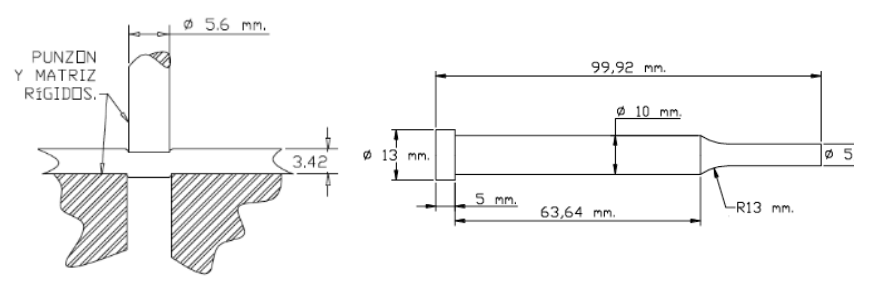

Figura 2. Geometría para evaluación de un proceso de corte

Fuente: Elaboración propia con datos de Boresi, Schmidt \& Sidebottom (1993)

La fuerza última de corte se obtiene de la solución matemática de la ecuación 1 (Beer y Johnston, 1993), conociendo los valores de área y esfuerzo cortante último del material AISI 1010 aplicando el círculo de Mohr que soporta el material. 


$$
\begin{aligned}
& \qquad F=A \tau_{u} \\
& \mathrm{~A}=\mathrm{L} * \mathrm{t}=60.167\left(\mathrm{~mm}^{2}\right) \\
& \mathrm{L}=2 \pi \mathrm{r}=2(3.1416)(2.8)=17.592(\mathrm{~mm}) \\
& \mathrm{t}=3.42(\mathrm{~mm}) \\
& \tau_{\mathrm{u}}=\frac{\sigma_{\mathrm{u}}}{2} 185(\mathrm{MPa}) \\
& \sigma_{\mathrm{u}}=370(\mathrm{MPa}) \\
& \mathrm{F}=11,065.94(\mathrm{~N})
\end{aligned}
$$

\subsection{Segundo paso; formular el problema de compresión en un punzón para conocer el esfuerzo máximo que puede soportar en carga axial.}

El punzón que se analiza es un punzón convencional de cambio rápido, con un cabezal, un cuerpo de sujeción al portapunzón, un cambio de sección y un cuerpo de corte como se muestra en la Figura 2, en este tipo de punzones, el punto más débil es el cambio de sección el cual es el que se somete al siguiente análisis de cálculo.

Con la fuerza de carga obtenida por el proceso de corte, $\mathrm{F}=$ $11,065.94(\mathrm{~N})$, se puede conocer el esfuerzo en el radio del cambio de sección del punzón, a este esfuerzo se le llama esfuerzo de carga axial, el esfuerzo en el cambio de sección puede observarse en la Tabla 1.

El esfuerzo por carga axial en el cambio de sección como se muestra en la Figura 2, se calcula por medio de la ecuación 2, (Shigley y Mischke, 1995), y si no sobrepasa el esfuerzo normal máximo del material con que se encuentra fabricado el punzón, por medio de la ecuación 3 (Shigley y Mischke, 1995), que es la fórmula del factor de seguridad de la teoría del esfuerzo normal máximo, se asegura que no fallará al momento de someterlo al proceso de corte.

$$
\sigma \frac{K}{A}
$$

$\mathrm{F}=11065.94(\mathrm{~N})$

$\mathrm{A}=\pi \mathrm{r}^{2}=3.1416(2.8)^{2}=24.63\left(\mathrm{~mm}^{2}\right)$

$\mathrm{K}_{t}=1$

El radio es su ficientemente grande para no maximizar el esfuerzo.

$\sigma_{y}=2,200(\mathrm{MPa})$

Esfuerzo de fluencia para un material D2.

$\sigma_{0}=449.28(\mathrm{MPa})$

$$
\eta=\frac{\sigma_{\mathrm{y}}}{\sigma_{0}}
$$

Por lo tanto el factor de seguridad es; $\eta=4.89$.

\subsection{Tercer paso; formular el problema de fatiga que se encuentra sometido el punzón para conocer el esfuerzo modificado por las cargas repetidas}

Sin embargo, los punzones se someten a esfuerzos de manera repetida a lo que pueden fracturarse por fatiga del material, por lo cual hay que calcular el esfuerzo límite de resistencia de fatiga, ecuación 4 (Shigley y Mischke, 1995), de la cual reduce el factor de seguridad del punzón que se calcula utilizando la ecuación 5, factor de seguridad de la teoría fatiga de Soderberg.

$$
\begin{aligned}
& \sigma_{\mathrm{m}}=\frac{\sigma_{\text {máx }}}{2}=224.64(\mathrm{MPa}) \\
& \sigma_{\mathrm{r}}=\sigma_{\text {máx }}=449.28(\mathrm{MPa}) \\
& \sigma_{\mathrm{a}}=\frac{\sigma_{\mathrm{r}}}{2}=224.64(\mathrm{MPa}) \\
& S_{e}=K_{a} K_{b} K_{c} K_{d} K_{e} S_{e}^{\prime}
\end{aligned}
$$

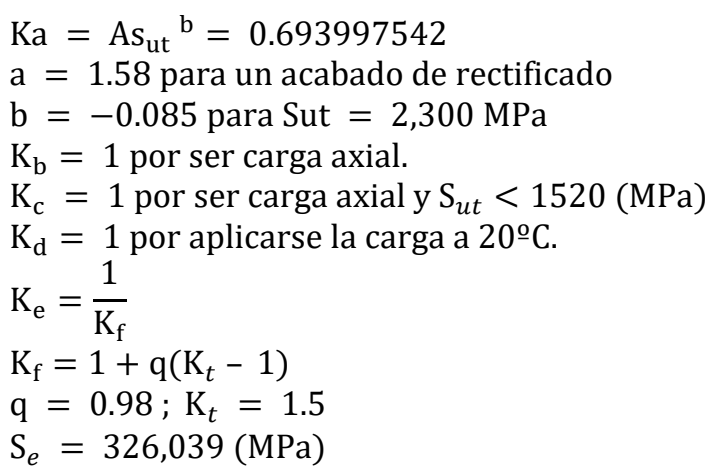

Por último, el factor de seguridad se reduce a 1.27, utilizando la teoría de fatiga de Soderberg. Cabe destacar que para materiales frágiles, comunmente para predecir la falla se ocupa la teoría del esfuerzo normal máximo; Coulomb-Mohr, fórmula 9 tomada de Shigley y Mischke (1995), la cual funcionaría a la perfección si los punzones y matrices de corte solo estuvieran sometidos a una carga constante, sin embrago, los punzones y matrices de corte son elementos sometidos a; maquinados superficiales, a un factor de tamaño, a un tipo de carga específica, a una temperatura de trabajo, así como otros factores que considera la teoría de fatiga de Soderberg.

Es así que para determinar la falla por fatiga, en esta investigación, se usa la teoría de Soderberg, ecuación 5, (Shigley y Mischke, 1995), es importante destacar que ninguna de las teorías, Soderber y Coulomb-Mohr, se contraponen, Soderberg se encuentra contenida en el polígono de falla por fricción interna (Coulomb-Mohr), Soderberg, es por tanto, un factor conservador que permite al diseñador calcular con mayor seguridad elementos mecánicos sin importar que sean de naturaleza fragil puesto que no solo se espera una falla del material, también se espera una falla por fatiga. El resultado obtenido quiere decir que el punzón solo puede soportar máximo un $27 \%$ de resistencia adicional en el material a cortar.

$$
\begin{array}{r}
\eta=\frac{1}{\frac{\sigma_{a}}{s_{e}}+\frac{\sigma_{m}}{s_{y t}}} \\
\eta=1.27
\end{array}
$$

Adicionalmente se puede generar una curva de esfuerzos como se muestra en la ecuación 6 (Shigley y Mischke, 1995), con dicha ecuación se puede conocer aproximadamente a los cuantos ciclos fallará el punzón, con los datos obtenidos se calcula que el punzón fallará al 1,000,106 ciclos de carga cuando el esfuerzo de fatiga se ha calculado a 326.039 (MPa).

$$
S f=a N b
$$




$$
\begin{aligned}
& \mathrm{a}=\frac{\left(0.9 \mathrm{~S}_{\mathrm{ut}}\right)^{2}}{\mathrm{~S}_{\mathrm{e}}}=114,228(\mathrm{MPa}) \\
& \mathrm{b}=\frac{\log \left(\frac{0.9 \mathrm{~S}_{\mathrm{ut}}}{\mathrm{S}_{\mathrm{e}}}\right)}{3}=-0,2675668
\end{aligned}
$$

$\mathrm{S}_{\mathrm{e}}=\mathrm{S}_{\mathrm{e}}^{\prime}=700(\mathrm{MPa}) ;$ en caso de $\mathrm{S}_{u t}>1,400(\mathrm{MPa})$

Por lo tanto la curva de ciclos contra esfuerzo

$$
\begin{array}{cc}
\text { Sf }=\text { 13,142.28 }\left(\mathrm{N}^{-0.26756688}\right) & \\
\text { Esfuerzo MPa. } & \text { Ciclos. } \\
13,142.28 & 1 \\
7,097.47 & 10 \\
3,832.98 & 100 \\
2,070.00 & 1,000 \\
1,117.90 & 10,000 \\
603.72 & 100,000 \\
326.03 & 1,000,106.30 \\
\text { límite a la fatiga } & \text { Ciclos } \\
\text { 176.07 } & 10,000,000 \\
\text { 3.4. Cuarto paso; formular el problema de esfuerzos de } \\
\text { contacto entre el punzón y el material a cortar }
\end{array}
$$

\subsection{Cuarto paso; formular el problema de esfuerzos de}

Adicionalmente, el contacto entre el punzón y el material a cortar también es crítico, el cálculo del esfuerzo de contacto permite determinar el valor que soporta el filo de corte, el cual es responsable de fracturas en el filo de corte del punzón. La Figura 2 representa el modelo de contacto entre el punzón y el material a cortar, y la ecuación 7 (Shigley y Mischke, 1995), esfuerzo de contacto, permite calcular el esfuerzo de contacto.

$$
\sigma_{c}=\frac{F}{2 \pi R^{3} \sqrt{R^{2}-r^{2}}}
$$

$$
\begin{aligned}
& \mathrm{F}=11,065.94(\mathrm{~N}) \\
& \mathrm{R}=2.8(\mathrm{~mm}) \\
& \mathrm{r}=0 \\
& \sigma_{\mathrm{c}}=224.64(\mathrm{MPa})
\end{aligned}
$$

\section{CONCLUSIONES DE LA EVALUACIÓN DE ESFUERZOS DE UN PUNZÓN DE CORTE DE METAL EN PROCESOS METALMECÁNICOS DE TROQUELADO}

La evaluación de los esfuerzos por diferentes métodos permite conocer con cuál de ellos la falla es prevenible.

Proceso de corte; indica que se requiere una fuerza de corte de $11,065.94(\mathrm{~N})$ para la geometría propuesta.

Carga axial del punzón a compresión; indica que el esfuerzo es de 449.28 (MPa) con un factor de seguridad de 4.89 veces, es decir que el punzón no fallará en el cambio de sección a menos que se aumente la resistencia del material a cortar en un $489 \%$.

Fatiga del punzón por cargas repetidas; indica que el punzón fallará en el cambio sección cerca de un millón de repeticiones y que la resistencia se reduce a $326.03(\mathrm{MPa})$ con un factor de seguridad de 1.27 , es decir que, si aumenta un $27 \%$ la resistencia al corte del material fallará en un corto plazo el punzón.

Esfuerzos de contacto entre superficies; indican que el filo del punzón solo soporta un esfuerzo de corte de 224.64 (MPa).

\section{MODELADO EN CAD-CAE}

El análisis en computadora se puede realizar en dos pasos, el primero es establecer los criterios básicos del CAE de condiciones de frontera, el segundo paso es establecer los criterios importantes del CAE de tipo de mallado y tipo de elementos seleccionados.

La aplicación utilizada es DesignSpace ${ }^{\mathrm{MR}}$, cabe destacar que este programa está diseñado para trabajar en el rango lineal y estructural de la mecánica de los materiales, por lo que los procesos de doblez y embutido no pueden ser modelados con este software, sin embargo, para cuestiones de aproximaciones resultó muy útil en CAE dado que es una aplicación de fácil aprendizaje y uso, además porque la aproximación que se realiza en este programa es para acercarse un momento antes del comportamiento no lineal del material, en otros programas especializados el diseador podrá utilizar herramientas no lineales.

El modelo paramétrico en tres dimensiones se puede generar en un programa como IronCAD ${ }^{\mathrm{MR}}$, que simula un ambiente de tercera dimensión en condiciones similares a las reales, de fácil interpretación y visualización, el modelado se realizó bajo las sugerencias de dibujo de Luzzader (1988), Chevalier (2009) y Jensen (1986).

La Figura 3a muestra un modelo en CAD básico, una aproximación con un CAD básico permite aproximaciones, con otros programas de modelado con mayores capacidades de modelado, se podrán controlar escalas para reducir cantidad de elementos, sin embargo para cuestiones de aproximación el modelo que se muestra en esta investigación es bastante cercano a la realidad, en conclusión, este modelo virtual es susceptible a evaluarse por elemento finito. Contiene el punzón de corte, la lámina a cortase y la matriz que completa la figura de corte.

Modelo de corte de lámina. Para idealizar el modelo de corte de material, se supone que tanto la matriz y el punzón son rígidos y no experimentan deformaciones, para lo cual se coloca la fuerza en la punta del punzón como se muestra en la Figura 3b.

Modelo del punzón. Para idealizar el análisis del punzón, se utiliza el mismo modelo descrito por la Figura $3 \mathrm{c}$ y el criterio para la aproximación del ambiente que supone un análisis a compresión teórico. 
El colocar una restricción adicional en la punta del punzón del modelo como se muestra en la Figura 3a, sirve para simular que la fuerza completa se aplica sobre el punzón, es notorio que ni el material ni la matriz serán afectados, solo que esta restricción, evita hacer un modelo exclusivo del punzón, también se coloca la fuerza en la base del punzón para simular la compresión axial del sistema.

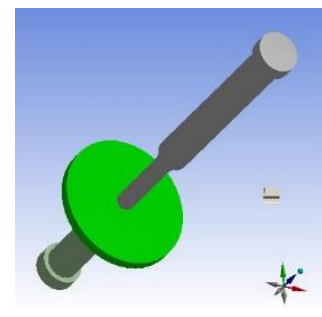

a) Modelo en CAD del proceso de corte b)

$$
\begin{aligned}
& \text { Modelo de corte } \\
& \text { de lámina }
\end{aligned}
$$

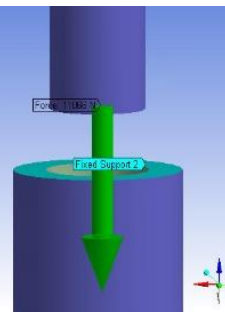

Figura 3. Modelado virtual Fuente: Elaboración propia

\subsection{Primer paso; criterios básicos del CAE de condiciones de frontera}

Modelo del contacto. Para suponer el contacto, no se utiliza directamente el modelo del proceso, se utiliza un modelo propuesto para analizar los esfuerzos.

La rigidez del material está acompañada de un soporte fijo en la parte inferior del modelo y un soporte de libertad axial del punzón.

Modelo del proceso. El analizar el modelo del proceso, sirve para encontrar los esfuerzos virtuales para los cuales no existen relaciones teóricas para analizarlos y que son valores aproximados a los verdaderos que suceden en el sistema y diferentes a los teóricos, para lo cual se suponen las condiciones de frontera.

La restricción fija en la base de la matriz indica que el movimiento está completamente restringido en todas direcciones. La restricción cilíndrica con libertad axial en la matriz y el punzón simulan la acción restringida en movimiento del modelo. La Fuerza indica la dirección de la misma y su magnitud en la cara seleccionada. El contacto sin deslizamiento y sin separación de los elementos es importante para simular la sujeción del modelo real que evitan la separación del material con la matriz y el punzón.

\subsection{Segundo paso; criterios importantes del CAE de tipo de mallado y tipo de elementos seleccionados}

Modelo de corte de lámina. En este modelo, se utiliza el mallado automático, el número de nodos fue 1,179 el número de elementos fue 158 con el elemento tetraedro.

Modelo del punzón. Este modelo también utiliza la selección de la convergencia como alternativa de refinado automático, para evitar conflictos de mallado en la computadora, el número de nodos fue 857 , el número de elementos fue 398 , con el elemento hexaedro.
Modelo del proceso. Para este modelo se refinan automáticamente los cuerpos, el número total de nodos fue 3,182 el número total de elementos fue 1,130 con el elemento hexaedro solamente en el área refinada, en el área visual de la figura se pueden ver tetrahedros en el exterior.

Modelo del contacto. En este modelo se combinan los mallados manuales y automáticos, debido a que el número de elementos es bajo y no provocan un error en el programa, se refinan los bordes de corte del punzón manualmente y se seleccionan las superficies de la lámina para asegurar la convergencia, el número de nodos fue 2,171 el número de elementos fue 1,251 con el elemento hexaedro.

\section{SOLUCIÓN E INTERPRETACIÓN DE RESULTADOS}

Un resultado virtual debe de interpretarse de manera lógica, porque no siempre manifiesta el valor o lugar exacto donde se espera que suceda una falla. Para poder explicar este punto, esta investigación se apoya en las gráficas visuales, estas indican con colores y etiquetas la magnitud donde ocurrirá la posible falla, otro apoyo es la convergencia matemática de error, cuyas gráficas indican la variación de la magnitud conforme el mallado es más fino, a continuación se muestran como interactúan estos dos conceptos entre si.

En este modelo, no existe convergencia utilizando la teoría del esfuerzo normal máximo, se supone que es porque la mayoría de los fenómenos de la naturaleza tienden a fallar a tensión y no a compresión y el programa DesignSpace ${ }^{\mathrm{MR}}$ esta diseñado para analizar solo este rango de los fenómenos utilizando la ecuación 8 (Shigley y Mischke, 1995), esfuerzo normal máximo para realizar sus procesos.

$$
\sigma_{0}=\sigma_{\max }=\left|\sigma_{1}\right|
$$

Por otra parte, se utiliza otra teoría aplicable al análisis de materiales frágiles, la teoría de Coulomb-Mohr, ecuación 9 (Shigley y Mischke, 1995), aunque esta teoría sea utilizada para materiales con diferentes valores de esfuerzo a la ruptura en compresión y a tensión, es una aproximación real y aplicable. El esfuerzo de Coulomb-Mohr consiste en alcanzar un cortante máximo preestablecido por diferentes estados de esfuerzo analizados, este esfuerzo se aplica a materiales frágiles Criterio de Coulomb-Mohr; $\sigma_{\mathrm{y}} \neq \sigma_{\mathrm{u}}$.

$$
\frac{\sigma_{1}}{S_{u t}}-\frac{\sigma_{3}}{S_{u t}}=\frac{1}{\eta}
$$

En este análisis $\sigma_{\mathrm{y}}=\sigma_{\mathrm{u}}$

la relación para el factor de seguridad es; $\eta=\frac{\sigma_{u}}{\sigma_{1}-\sigma_{3}}$

\section{CONFIABILIDAD DEL ANÁLISIS VIRTUAL}

La interpretación de la iteración, son aproximaciones matemáticas internas del CAE, con la finalidad de que por medio de algún método iterativo se reduzca el error, depende del algoritmo de programación del programa, asegura la convergencia matemática del cálculo y contribuye a la reducción del cálculo. 
En esta sección de confiablidad se agregan la convergencia de los procesos que se realizaron en la etapa de CAE, corte de material, resultados del punzón convencionalmente y el cálculo a fatiga, así como el contacto entre el punzón y el material.

Resultados de corte de material. La convergencia sucede de la $3^{\mathrm{a}}$ a la $5^{\mathrm{a}}$ iteración y se espera la superficie del material, véase las Figuras 4a y 4b.

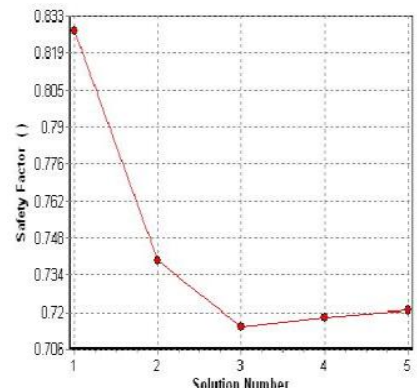

a) Convergencia del factor de seguridad de la teoría del esfuerzo cortante máximo del proceso

Figura 4. Convergencia del factor de seguridad esfuerzo cortante máximo Fuente: Elaboración propia

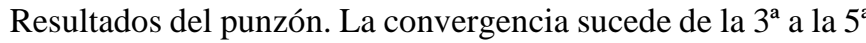
iteración y se espera en el borde del material, aquí la influencia del esfuerzo de contacto es notoria, ya que se convierte en el esfuerzo crítico para un punzón, véase las Figuras 5a y 5b.

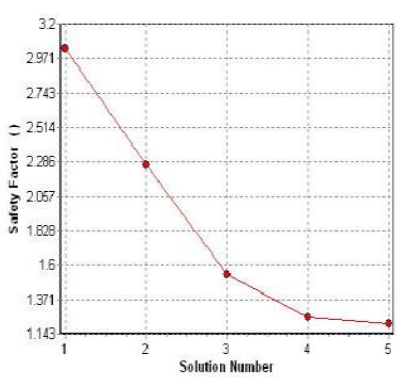

a) Convergencia del factor de seguridad de la teoría del esfuerzo de Coulomb-Mohr

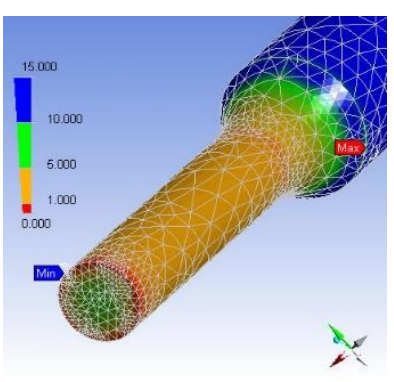

b) Factor de seguridad de la teoría del esfuerzo de Coulomb-Mohr
Figura 5. Convergencia del factor de seguridad de la teoría del esfuerzo de Coulomb-Moh

Fuente: Elaboración propia

Resultados a fatiga. La convergencia sucede de la $3^{\mathrm{a}}$ a la $5^{\mathrm{a}}$ iteración y se espera en el borde del material, aquí la influencia del esfuerzo de contacto también es notoria, ya que por ser el esfuerzo crítico para un punzón, la fatiga se programa para que se analice utilizando el esfuerzo normal en Z, como se realizó en la suposición del esfuerzo de contacto.

Modelo del contacto. La convergencia sucede en la $2^{\mathrm{a}}$ iteración y se espera la falla en el borde del punzón.

La Tabla 1 muestra los valores de cada análisis convencional, contra los análisis CAE.

El CAE, ante el análisis del esfuerzo cortante máximo, es menor en $3 \%$, es un valor muy útil porque calcula con confianza $3 \%$ por encima de lo que se espera en un análisis convencional.

El CAE ante la teoría de Coulomb-Mohr, es menor en un $11.86 \%$, es un valor muy útil porque calcula con confianza $11.86 \%$ por encima de lo que se espera en un análisis convencional.

El CAE ante la teoría de fatiga de Soderberg, es mayor en un $23.80 \%$, es un valor poco confiable porque ofrece un $23.80 \%$ porque eleva el valor esperado en un análisis convencional.

El CAE ante el contacto, es mayor en un $26.05 \%$, es un valor poco confiable porque ofrece un $26.05 \%$ porque eleva el valor esperado en un análisis convencional.

Tabla 1: Confiabilidad de análisis virtuales contra teóricos en el proceso de

\begin{tabular}{|c|c|c|c|c|}
\hline \multirow[b]{3}{*}{ Teoría. } & \multicolumn{4}{|c|}{ Factor de seguridad. } \\
\hline & \multirow[b]{2}{*}{ Teórico } & \multicolumn{2}{|c|}{ Virtual. } & \multirow{2}{*}{$\begin{array}{l}\% \text { de } \\
\text { error } \\
\text { teórico }\end{array}$} \\
\hline & & F.S. & $\begin{array}{c}\% \text { de } \\
\text { error de } \\
\text { iteración }\end{array}$ & \\
\hline \multicolumn{5}{|c|}{ Lámina. } \\
\hline $\begin{array}{l}\text { Teoría del esfuerzo } \\
\text { cortante máximo }\end{array}$ & 1 & 0.97 & $0.34 \%$ & $-3.00 \%$ \\
\hline \multicolumn{5}{|c|}{ Punzón. } \\
\hline $\begin{array}{l}\text { Teoría de } \\
\text { Coulomb-Mohr }\end{array}$ & 4.89 & 4.31 & $3.60 \%$ & $-11.86 \%$ \\
\hline $\begin{array}{l}\text { Teoría de fatiga de } \\
\text { Soderberg }\end{array}$ & 1.26 & 1.56 & $6.42 \%$ & $+23.80 \%$ \\
\hline \multicolumn{5}{|c|}{ Contacto. } \\
\hline Esfuerzo axial & 224.64 & 283.15 & $3.79 \%$ & $+26.05 \%$ \\
\hline
\end{tabular}

Fuente: Elaboración propia / cálculo matemático * Todos los modelos convergieron en la $5^{\mathrm{a}}$ iteración.

Los resultados de la comprobación de los resultados teóricos contra resultados reales utilizando probetas en ensayos destructivos no se reportan en este documento por ser confidenciales, sin embargo, se presentan los resultados convencionales, Tabla 1, para que analistas de ingeniería, estudiantes y profesores utilicen la información de este documento con fines didácticos o consulta, los cuales les pueden ser útiles para generar su propia línea de investigación.

Aunque los resultados de los ensayos destructivos no se muestran en este documento por considerarse confidenciales, se muestra una fotografía, Figura 6 , en la que se puede observar claramente el tipo de falla que sucede al someter al trabajo contínuo a los punzones y matrices, en dicha fotografía se observa que la falla ocurre en el filo de corte, lo cual es congruente con la Figura 5 generada virtualmente.

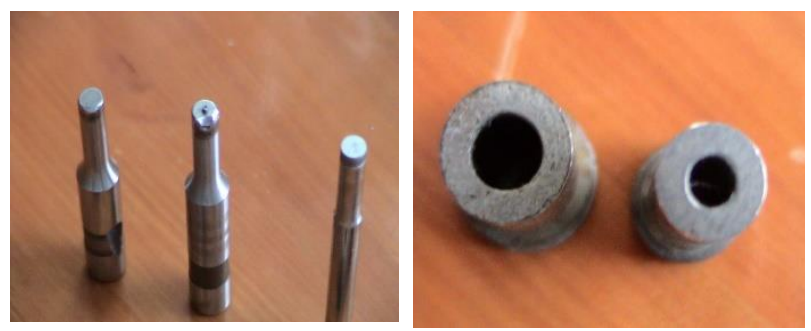

Figura 6. Fallas en punzones y Fallas en matrices Fuente: Elaboración propia 
Por último, se recomienda que una vez que se ha dominado el proceso de análisis virtual, como el que muestra este documento, se realicen los ensayos destructivos y no destructivos respectivos, el lector puede consultar algunos estudios de comparación que contrastan la simulación en CAD-CAE contra resultados de ensayos destructivos como el realizado por Castaño-Urrego, Lopez-Perez, Forero-Rubiano y Tlapanco-Rios (2020) llamado "Método de comparación de resultados de modelado CAD-CAE contra probetas de ensayo destructivo" en dicho estudio una empresa de transporte solicitó analizar un problema de ensamble con ayuda virtual, el resultado que se muestra en dicho estudio muestra un error del cálculo virtual CAD-CAE y los ensayos destructivos que varía entre los valores de $1.54 \%$ a $2.08 \%$.

Por otra parte el diseñador que esté siguiendo la metodología del TRL, puede estar seguro que los resultados son iterativos, es decir que requerirá de validar sus análisis con pruebas y ensayos desde la etapa TRL 1 hasta TRL 5 como se sugiere en la tabla 5 , que se refiere al proceso sugerido para utilizar la Norma Oficial Mexicana NMX-GT-004-IMNC-2012; es decir el trabajo de modelado no termina con comprobación de resultados teóricos por medio de CAD-CAE, pues deberá seguir paso a paso el desarrollo tecnológico generado previamente para concluir con la innovación esperada.

\section{CONCLUSIONES DEL MODELADO CAD-CAE}

Estas conclusiones son válidas porque con el uso de estas aproximaciones se logró diseñar elementos de troquelado para la industria que solicitó el análisis, la empresa validó los resultados utilizando los mismos criterios de CAD-CAE en la fabricación de otros punzones y matrices con contornos no convencionales, Figura 7. Los resultados fueron satisfactorios, validados y de alto valor para la empresa.

Cabe destacar que esta investigación es una propuesta y utiliza una estrategia que el diseñador fue alternando con el software que se le proporcionó. Cada diseñador es libre de proponer estrategias diferentes y adecuadas con el uso de diferentes programas que estén a su alcance.

En la actualidad, el proceso de diseño de un troquel puede ser acompañado por el análisis por elemento finito de los elementos que lo componen debido a que el análisis modifica sustancialmente el diseño y que es una herramienta de apoyo para conocer de manera temprana si lo que se está diseñando cumple con las características de ingeniería y en su caso mejorarlo asegurando que el sistema funcionará con factores de seguridad.

En un modelo complejo no existen formulaciones convencionales para el análisis, por lo que es necesario recurrir a una formulación virtual como el CAD-CAE. En la Figura 7 se puede observar un modelo complejo, en la parte inferior o base se encuentra la matriz, la placa rectangular es la lámina y el elemento superior es el punzón.

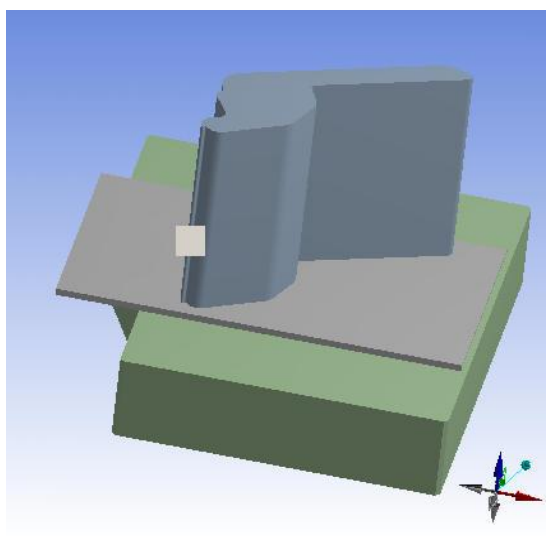

Figura 7. Modelo de configuración compleja Fuente: Elaboración propia

La Figura 7 puede ser sometida a un análisis CAE, si se utiliza el mismo método planteado en esta investigación, y al menos se tiene que contemplar que:

- El CAE ante el análisis del esfuerzo cortante máximo, estará $3 \%$ por debajo de lo que se espera en un análisis convencional.

- El CAE ante la teoría de Coulomb-Mohr, estará $11.86 \%$ por debajo de lo que se espera en un análisis convencional.

- El CAE ante la teoría de fatiga de Soderberg, incrementará un $23.80 \%$ el valor esperado en un análisis convencional.

- El CAE ante el contacto, incrementará $26.05 \%$ el valor esperado en un análisis convencional.

\section{CONCLUSIONES}

La estrategia de innovación de producto en procesos de diseño mecánico finaliza al responder las preguntas correspondientes al nivel TRL 1 como lo solicita la Norma Oficial Mexicana NMX-GT-004-IMNC-2012.

Para este caso, las preguntas que se pueden responder son las siguientes: ¿Finalizó con la investigación básica de su idea? La respuesta es una certeza por lo que se puede responder con un si, y la pregunta ¿Identificó principios de investigación básica que pudieran trasladarse en principios nuevos que puedan ser utilizados en nuevas tecnologías? La respuesta es una certeza por lo que se puede responder con un si. Estas respuestas son el Inicio del Proceso como se sugiere en la Tabla 3.

Por último, el aplicar metódicamente el modelo nacional de gestión de tecnología (NMX-GT-004-IMNC-2012) es una herramienta para la planeación estratégica de la innovación, además de que permite ubicar las actividades de innovación. El desarrollo de cada uno de los TRL permite generar bases de datos que reducen la incertidumbre tecnológica para otros proyectos tecnológicos. En este caso se difunde por medio de la publicación de esta investigación un TRL 1 que pueda servir de ejemplo en el diseño, pero más importante es el difundir ejemplos de proyectos TRL en todos los niveles, con fines metodológicos y didácticos, que reduzcan la subjetividad del 
desarrollo tecnológico, la Tabla 2 es la gúia principal para el seguimiento del TRL, y la Tabla 3 es un diagrama de flujo basado en la Norma Oficial Mexicana NMX-GT-004-IMNC2012, la cual es una propuesta para la toma de decisiones que conduzca al logro total de lo nueve niveles del TRL.

Los conceptos de la tabla 4 que se refieren a la Norma Oficial Mexicana NMX-GT-004-IMNC-2012, los describe el CONACYT y se transcriben a continuación:

"Technology Readiness Level, TRL: Es una escala de medición usada para evaluar o medir el nivel de madurez de una tecnología particular. Cada proyecto tecnológico es evaluado frente a los parámetros de cada nivel tecnológico y es asignado a una clasificación basada en el progreso del proyecto.

Investigación y Desarrollo Experimental (I+D): Es una actividad de innovación tecnológica puede ser llevada a cabo en diferentes fases del proceso de innovación, siendo utilizada no sólo como la fuente de ideas creadoras sino también para resolver los problemas que pueden surgir en cualquier fase de dicho proceso hasta su culminación. Comprende la investigación básica, la investigación aplicada y el desarrollo experimental.

La investigación básica consiste en trabajos experimentales o teóricos que se emprenden principalmente para obtener nuevos conocimientos acerca de los fundamentos de los fenómenos y hechos observables, sin pensar en darles ninguna aplicación o utilización determinada.

La investigación aplicada consiste también en trabajos originales realizados para adquirir nuevos conocimientos; sin embargo, está dirigida fundamentalmente hacia un objetivo práctico específico.

El desarrollo experimental consiste en trabajos sistemáticos que aprovechan los conocimientos existentes obtenidos de la investigación y/o la experiencia práctica, y está dirigido a la producción de nuevos materiales, productos o dispositivos; a la puesta en marcha de nuevos procesos, sistemas y servicios, o a la mejora sustancial de los ya existentes Fuente: Manual de Frascati (2002) OCDE, Propuesta de Norma Práctica para Encuestas de Investigación y Desarrollo Experimental.

Innovación: Es la introducción al mercado de un producto (bien o servicio), proceso, método de comercialización o método organizacional nuevo o significativamente mejorado, por parte de una organización. (Manual de Oslo, 2005, OCDE, Tercera edición.) Observe que sus principales características son que es algo nuevo, único y comercializable.

Innovación tecnológica: Innovación que se distingue por una mejora o novedad en las características del desempeño de los productos o servicios, y su aplicabilidad en la práctica dependerá del grado en que dichas características y su grado de novedad sean un factor importante en las ventas de una empresa o industria concerniente. (Manual de Oslo, 2005, OCDE, Tercera edición.)
Desarrollo tecnológico: Desarrollo Tecnológico. Uso sistemático del conocimiento y la investigación dirigidos hacia la producción de materiales, dispositivos, sistemas o métodos incluyendo el diseño, desarrollo, mejora de prototipos, procesos, productos, servicios o modelos organizativos (LCTI).

Propiedad intelectual: Conjunto de derechos de carácter exclusivo que otorga el Estado, por tiempo determinado, a las personas físicas o morales que han realizado creaciones intelectuales abarcando las obras contempladas por la Propiedad Industrial, Derechos de Obtentor y el Derecho de Autor. (NMX-GT-001-IMNC-2007)

Pruebas: Se refiere a todas las actividades que sean requeridas para validar y demostrar la funcionalidad de los componentes así como su aplicación.

Principios básicos: Es aquello que sustenta, respalda y da validez a la idea y traza la línea de investigación.

Validación comercial / Quick Look: Poder identificar-validar, a través del contacto con fuentes primarias directas, las secciones del CANVAS relacionadas con los segmentos de clientes, mercado y propuesta de valor que agrega la oferta tecnológica.

El Quick Look contiene lo siguiente, utilizando como referencia fuentes secundarias como marco referencial, pero principalmente de fuente primarias (de preferencia al menos 100 entrevistas con actores clave de la industria en cuestión): Descripción de la tecnología, beneficios potenciales, mercados potenciales para la comercialización, interés de los mercados, estado que guarda el desarrollo tecnológico, proceso de protección intelectual, tecnologías que compiten y competidores "análisis de benchmarking", identificación de barreras de entrada al mercado, recomendaciones, medición del nivel potencial de comercialización, realizar entrevistas con expertos de mercado y de transferencia de tecnología y documentación de entrevistas (Internet, presenciales, telefónicas, grupos de enfoque, etc.).

Ambiente operativo real: Pruebas y validaciones con usuarios reales y potenciales." 
Tabla 2: Niveles TRL, Norma Oficial Mexicana NMX-GT-004-IMNC-2012

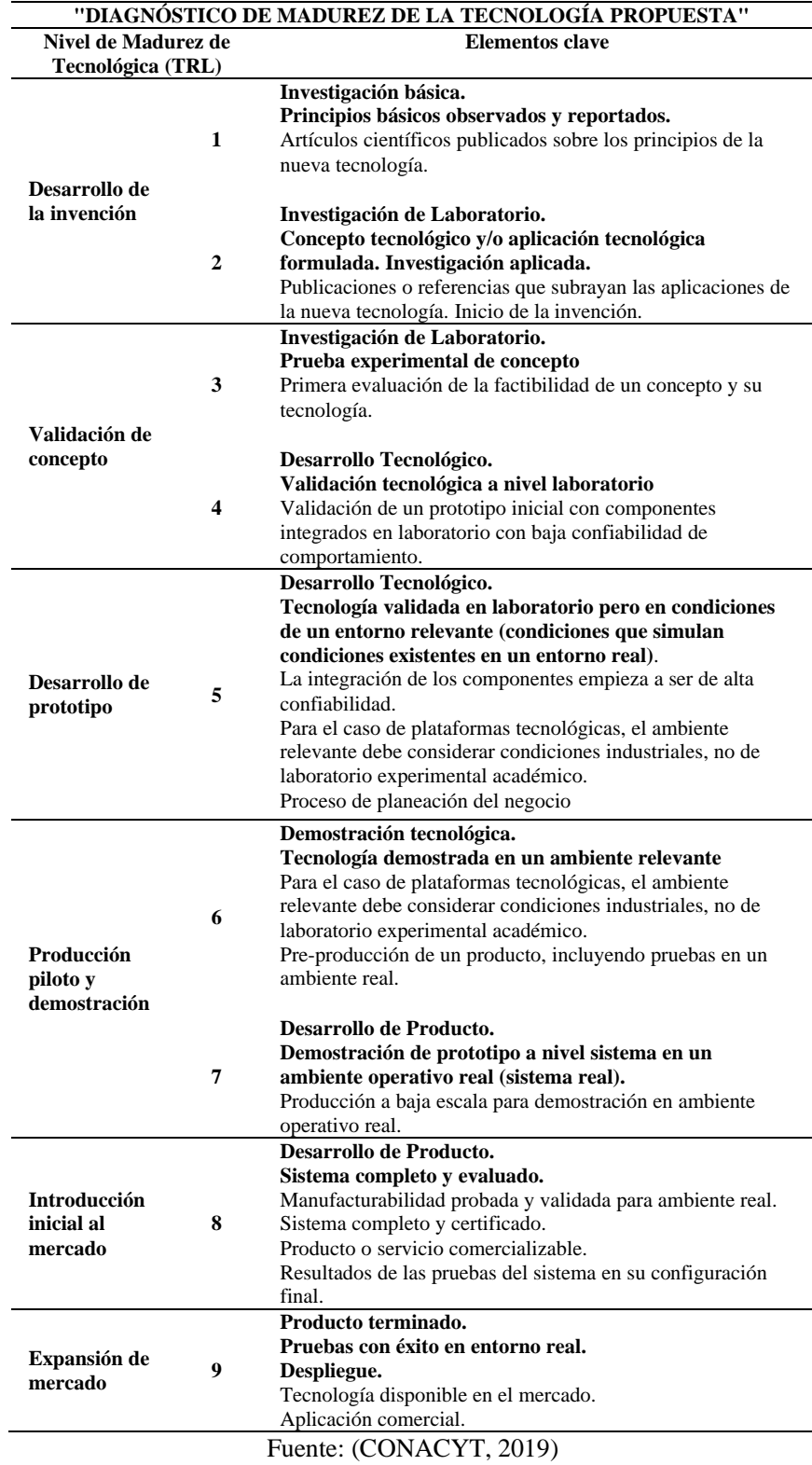

Algunos otros estudios que se pueden consultar para una mejor comprensión del TRL son los realizados por David Moorhouse (2002) en su artículo "Detailed Definitions and Guidance for Application of Technology Readiness Levels", en el cual describe detalladamente la terminología del TRL de los niveles 1 al 7, rango que el autor considerada como el rango de desarrollo tecnológico, y la finalidad de que quien consulte el artículo de Moorhouse le sirva de ayuda en la toma de decisiones gerenciales. Otro estudio como el de John Mankins (2009) en su artículo "Technology readiness assessments: A retrospective" nos hace una remembranza histórica de la formación del TRL dentro de la NASA, dentro del Departamento de Defensa de los Estados Unidos de América y el Congreso de la Unión, el recorrido lo hace a través de los últimos 30 años desde la década de 1970, otro artículo como el de Don Clausing y Maurice Holmes (2010) en su artículo “Technology Readiness" explora el proceso de la innovacoón, es decir de como utilizar el TRL como parte de un método para que la inversión económica en un proyecto sea altamente confiable y de muy bajo riesgo de inversión en el desarrollo tecnológico.
Por último, se muestra la propuesta de un diagrama de flujo respecto a la Norma Oficial Mexicana analizando la propuesta de la misma norma provenientes de la Tabla 3.

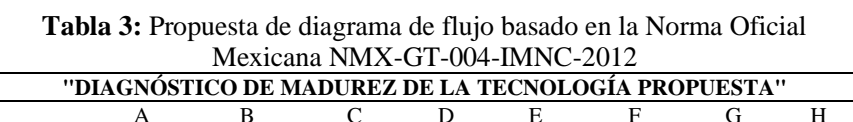
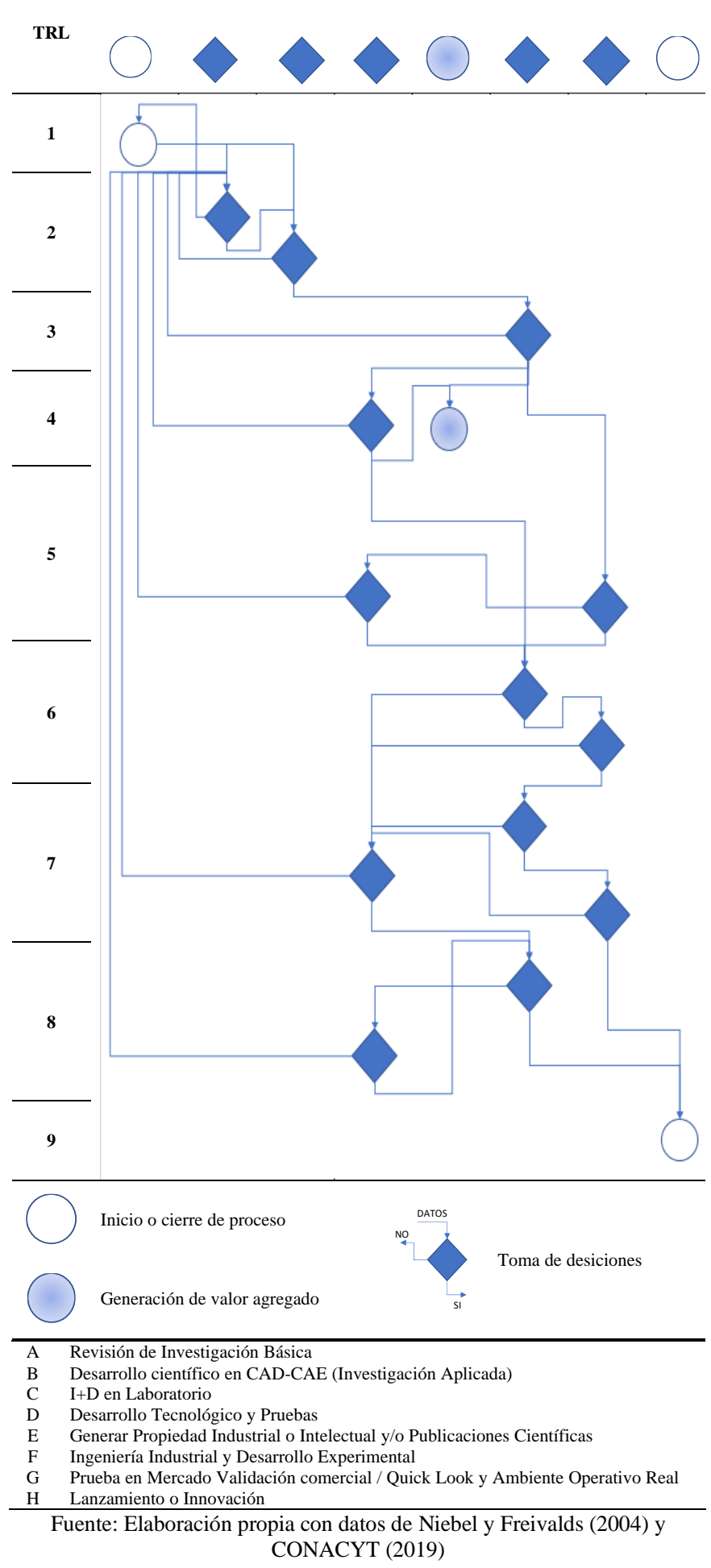

\section{AGRADECIMIENTOS}

A la Universidad de Guanajuato y al Tecnológico Nacional de México Instituto Tecnológico de Celaya por los equipos de Profesores de Ingeniería Mecánica, en especial al M. I. Raúl Lesso Arroyo (1965-2019). 


\section{REFERENCIAS}

Chevalier, A. (2009). Dibujo Industrial. México: Limusa.

Consejo Nacional de Ciencia y Tecnología. (11 de Abril de 2019). CONACyT. Obtenido de Convocatoria 2017-02 "CONACYT-SENERSustentabilidad Energética-Institutional Links": https://www.conacyt.gob.mx/index.php/sni/convocatoriasconacyt/convocatorias-fondos-sectorialesconstituidos/convocatoria-sener-conacyt-sustentabilidadenergetica/convocatorias-cerradas-sener-conacyt-sustentabilidadenergetica/convocatoria-2017-02-conacyt

Luzzader, W. J. (1988). Fundamentos de Dibujo en Ingeniería. Naucalpan de Juárez: Pretince Hall.

Beer, F. P., y Johnston, Jr, E. R. (1993). Mecánica de Materiales. Santafé de Bogotá: McGraw Hill Interamericana S. A.

Boresi, A. P., Schmidt, R. J., y Sidebottom, O. M. (1993). Advanced Mechanics of Materials. New York: John Wiley \& Sons. Inc.

Castaño-Urrego, C. A., Lopez-Perez, J. R., Forero-Rubiano, R. A., y Tlapanco-Rios, E. I. (2020). Método de comparación de resultados de modelado CAD-CAE contra probetas de ensayo destructivo. Acta Universitaria 30. doi. http://doi.org/10.15174.au.2020.2668.

Clausing, D. y Holmes, M. (2010). Technology Readiness. ResearchTechnology Management, Volume 53, Pages 52-59. doi. https://doi.org/10.1080/08956308.2010.11657640.

Garcia Martinez, J. G., y Münch Galindo, L. (1992). Fundamentos de Administración. Trillas.

Guinet, J. (1995). National Systems for Financing Innovation. Paris: Organisation For Economic Co-operation and Development.

Hidalgo, A. (2006). Mecanismos de transferencia de tecnología y propiedad industrial entre la Universidad, los Organismos Públicos de Investigación y Empresas. Madrid: EOI ESCUELA DE ORGANIZACIÓN INDUSTRIAL.

Industrial Press Inc. (1992). Machinery's Handbook Twenty-Fourth Edition. New York: Industrial Press Inc.

Jensen, M. (1986). Fundamentos de Dibujo Mecánico: McGraw Hill.

Ministerio de Industria, Energía y Turismo. (2014). Aplicaciones de le Teoría de Juegos a la Economía Industrial: Economía Industrial.

Mankins, J. C. (2009). Technology readiness assessments: A retrospective. Acta Astronautica, Vol. 65 (9-10), p. 1216-1223. doi. https://doi.org/10.1016/j.actaastro.2009.03.058.

Moorhouse, David. (2002). Detailed Definitions and Guidance for Application of Technology Readiness Levels. Journal of Aircraft, Volume 39 (1), p. 190-192. doi. https://doi.org/10.2514/2.2916.

Mott, R. L. (1995). Diseño de elementos de máquinas. Naucalpan de Juárez: Pretince Hall Hispanoamericana S. A

Niebel, B. W., y Freivalds, A. (2004). Ingeniería Industrial, Métodos, Estándares y Diseño del Trabajo. México, D. F.: Alfaomega.

Shigley, J. E., y Mischke, C. R. (1995). Diseño en Ingeniería Mecánica: McGraw Hill.

Society Of Manufacturing Engineers. (1990). Die Design Handbook. Dearborn: Society of Manufacturing Engineers.
Timoshenko, S. P., y Goddier, J. N. (1987). Theory of Elasticity: McGraw Hill

Uddeholm. (2003). AISI D2 Cold Work Tool Steel: Steels for Cold Work Tooling

\section{BIOGRAFÍAS}

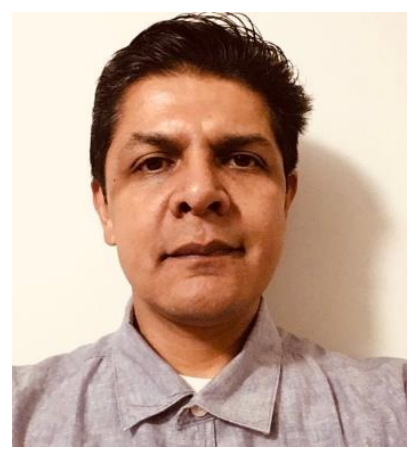

Dr. Ernesto Isaac Tlapanco Rios, desarrolla la línea de investigación de Sistemas de Innovación, Transferencia de Tecnología y Emprendimiento de Base Tecnológica. Tiene en proceso 3 títulos de Propiedad Industrial, 1 publicación indexada, 1 capítulo de libro. Director de 4 tesistas. Responsable Técnico en 2 proyectos de investigación. Es Doctor en Ciencias del Desarrollo Regional, Maestro en Gestión Administrativa e Ingeniero Mecánico con especialidad en Diseño. Es Profesor Investigador en la Universidad de Guanajuato. De 1999 a 2009 laboró aplicando la Ingeniería en Procesos, Proyectos y Diseño para el grupo Uniko, Acros-Whirlpool, General Mills y Arbomex.

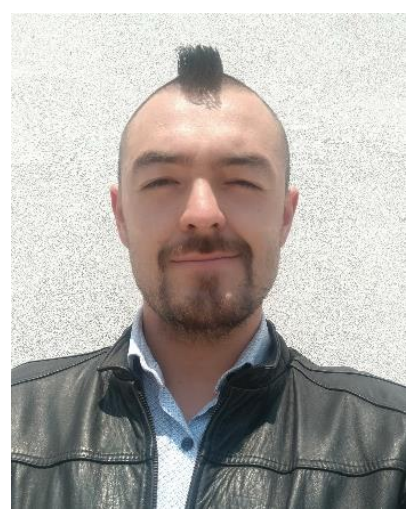

Carlos Andrés Castaño Urrego, nacido en Bogotá (Colombia) en 1994. Cursó los estudios primarios y secundarios en Colegio Militar Caldas (Colombia), sus estudios de ingeniero mecánico en la universidad Santo Tomás (Colombia). Estudiante de la maestría en ciencias de la Administración de Tecnologías en la universidad de Guanajuato (México) desde el 2020. Ejerció como diseñador en Siberia, Cundinamarca (Colombia). Profesor nivel licenciatura en la universidad de Guanajuato. 